OPEN ACCESS

Edited by:

R. Thomas Jagoe,

McGill University, Canada

Reviewed by:

Charlotte K. Callaghan, Trinity College Dublin, Ireland

Loredana Bergandi,

University of Turin, Italy

*Correspondence:

Hélène Castel

helene.castel@univ-rouen.fr

Specialty section:

This article was submitted to Pharmacology of Anti-Cancer Drugs,

a section of the journal

Frontiers in Pharmacology

Received: 15 November 2016 Accepted: 06 March 2017

Published: 21 March 2017

Citation:

Castel $H$, Denouel A, Lange M, Tonon M-C, Dubois M and Joly F (2017) Biomarkers Associated with

Cognitive Impairment in Treated Cancer Patients: Potential Predisposition and Risk Factors. Front. Pharmacol. 8:138 doi: 10.3389/fphar.2017.00138

\title{
Biomarkers Associated with Cognitive Impairment in Treated Cancer Patients: Potential Predisposition and Risk Factors
}

\begin{abstract}
Hélène Castel ${ }^{1,2,3 *}$, Angeline Denouel ${ }^{4}$, Marie Lange ${ }^{4,5}$, Marie-Christine Tonon ${ }^{1,2,3}$, Martine Dubois ${ }^{1,2,3}$ and Florence Joly ${ }^{3,4,5,6}$

${ }^{1}$ Laboratory of Neuronal and Neuroendocrine Differentiation and Communication, Institut National de la Santé et de la Recherche Médicale, DC2N, Normandie University, Rouen, France, ${ }^{2}$ Institute for Research and Innovation in Biomedicine, Rouen, France, ${ }^{3}$ Cancer and Cognition Platform, Ligue Nationale Contre le Cancer, Caen, France, ${ }^{4}$ Institut National de la Santé et de la Recherche Médicale, U1086, Caen, France, ${ }^{5}$ Medical Oncology Department, Centre François Baclesse,

Caen, France, ${ }^{6}$ Medical Oncology, University Hospital Center, Caen, France
\end{abstract}

Purpose: Cognitive impairment in cancer patients induced, at least in part, by treatment are frequently observed and likely have negative impacts on patient quality of life. Such cognitive dysfunctions can affect attention, executive functions, and memory and processing speed, can persist after treatment, and their exact causes remain unclear. The aim of this review was to create an inventory and analysis of clinical studies evaluating biological markers and risk factors for cognitive decline in cancer patients before, during, or after therapy. The ultimate objectives were to identify robust markers and to determine what further research is required to develop original biological markers to enable prevention or adapted treatment management of patients at risk.

Method: This review was guided by the PRISMA statement and included a search strategy focused on three components: "cognition disorders," "predictive factors"/"biological markers," and "neoplasms," searched in PubMed since 2005, with exclusion criteria concerning brain tumors, brain therapy, and imaging or animal studies.

Results: Twenty-three studies meeting the criteria were analyzed. Potential associations/correlations were identified between cognitive impairments and specific circulating factors, cerebral spinal fluid constituents, and genetic polymorphisms at baseline, during, and at the end of treatment in cancer populations. The most significant results were associations between cognitive dysfunctions and genetic polymorphisms, including APOE-4 and COMT-Val; increased plasma levels of the pro-inflammatory cytokine, IL-6; anemia; and hemoglobin levels during chemotherapy. Plasma levels of specific hormones of the hypothalamo-pituitary-adrenal axis are also modified by treatment.

Discussion: It is recognized in the field of cancer cognition that cancer and comorbidities, as well as chemotherapy and hormone therapy, can cause persistent cognitive dysfunction. A number of biological circulating factors and genetic polymorphisms, can predispose to the development of cognitive disorders. However, 
many predictive factors remain unproven and discordant findings are frequently reported, warranting additional clinical and preclinical longitudinal cohort studies, with goals of better characterization of potential biomarkers and identification of patient populations at risk and/or particularly deleterious treatments. Research should focus on prevention and personalized cancer management, to improve the daily lives, autonomy, and return to work of patients.

Keywords: cognitive disorders, biological markers, predictive factors, cancer, chemotherapy

\section{INTRODUCTION}

There have been improvements in the efficacy of cancer treatments, and also in the management of side effects and patient care over the last decade. However, cancer treatments, most often chemotherapy, may induce side effects on the bone marrow, heart, cardiac, or digestive system and often cause nausea, alopecia, or even cognitive impairments Ahles (2012). Chemotherapy can cross the blood brain barrier (BBB) and cause brain damage (Cheung et al., 2015; Wang et al., 2015), which could explain cognitive impairments, including of concentration, memory, executive functions, and processing speed, symptoms often referred to as "chemofog" or "chemobrain" (Vardy et al., 2008; Joly et al., 2015). These cognitive disorders can have major consequences on patient quality of life, return to work, or autonomy, and thus represent a major public health issue which requires investigation.

To identify and characterize subgroups of patients at risk of cognitive impairment induced by cancer and its treatment, and to adapt patient treatment, it is essential to discover biological factors mediating cognitive problems and/or risk factors, such as genetic polymorphisms, inflammatory indicators, or blood biomarkers (Kesler et al., 2013; Wang et al., 2015). Some biomarkers, that are either predictive of risk or produced in response to treatment or the cancer itself, can be relatively easily measured by blood sampling before, during, and after management of the cancer. Such biological predictive factors may also correlate with cerebral imaging, to provide information about brain structure and volume changes involved in cognitive impairment (Wang et al., 2015).

The objective of this review was to establish a summary of original articles published since 2005, including all biological predictive factors of cognitive changes in cancer patients, particularly after cancer treatment. Moreover, we discuss the

\footnotetext{
Abbreviations: AC/CAF, Cyclophosphamide/cyclophosphamide plus fluorouracil; ALL, Acute lymphoblastic leukemia; APOE, Apolipoprotein E; A $\beta 40$ and A $\beta 42$, amyloid- $\beta$ peptides 40 and 42; CMF, Cyclophosphamide, methotrexate, and fluorouracil; COMT, Catechol-O-methyltransferase; CRP, C-reactive protein; CSF, Cerebrospinal fluid; CT, Chemotherapy; DNA, Deoxyribonucleic acid; Dox, Doxorubicin; ELISA, Enzyme-linked immunosorbent assay; EPO, Erythropoietin; GnRH, Gonadotropin-releasing hormone; Hb, Hemoglobin; IL, Interleukin; INF $\gamma$, Interferon $\gamma$; LPC, Lysophosphatidylcholine; MCP-1, Monocyte chemoattractant protein 1; MMSE, Mini-mental State Examination; MTHFR, 5,10-methylenetetrahydrofolate; mRCC, Metastatic renal cell cancer; pNF-H, Phosphorylated neurofilament subunit H; SM, Sphingomyelin; TNF $\alpha$, Tumor necrosis factor-alpha; TNF-RII, Tumor necrosis factor-receptor type II; VEGF, Vascular endothelial growth factor; VEGFR TKI, Tyrosine kinase VEGF receptor; RT, Radiotherapy.
}

limitations of these studies, concerning their different types, methods, results, and interpretation.

\section{METHODS USED FOR INFORMATION STRATIFICATION}

Articles were retrieved from PubMed using the following key words:

- MeSH terms: "cognition disorders," "neurotoxicity syndromes," "biological markers," "prognosis," "biological factors"

- PubMed terms: "predictive factors," "cancer," "chemobrain,” "chemofog," "cognitive dysfunction," "cognitive impairments"

This review was guided by the PRISMA statement and used a search strategy focused on three components: "cognition disorders," "predictive factors"/"biological markers," and "neoplasms," searched in PubMed (with MeSH and PubMed terms). Original studies since 2005 were included, regardless of type (i.e., cross-sectional and longitudinal, randomized, and non-randomized, single center and multicenter). Selection was not based on cancer type; mainly acute lymphoblastic leukemia (ALL), acute myeloid leukemia (AML), myelodysplastic syndrome (MDS), breast, lung, prostate, and differentiated thyroid carcinoma were included; however, brain tumors and cancers involving brain metastasis were excluded, because of their mass effects and potential consequences of surgery/resection on the brain, which are likely to directly impact cognitive function (Table 1). Moreover, all types of cancer treatments were included, except encephalic radiotherapy, which can have direct effects on brain function, edema, and cognition (Table 1). Studies for which predictive factors were cerebral and/or imaging parameters (magnetic resonance imaging/hippocampal volume or metabolic activity) or that did not address one or more of the three components, cancer, cognition, or biological mechanisms, were also excluded, along with clinical, physiopathological, and psychological parameters. Only human studies were included, thus preclinical animal studies were not taken into account.

The first exclusion criterion, evaluated by reading abstracts, was the absence of at least one of the three domains, i.e., "cognition disorders," "predictive factors,"/"biological markers," and "neoplasms." Between 2005 and 2015, 65 studies at least partly covered the topic under investigation. Other exclusion criteria, determined by reading entire papers, concerned studies of brain tumors or cranial radiotherapy, or the absence of 
TABLE 1 | Main antitumor treatments and their mechanisms of action reported within the 23 selected publications that can be linked to modified biological factors and cognitive dysfunctions.

\begin{tabular}{|c|c|c|}
\hline Cancer type & Treatment & Mechanisms of action \\
\hline \multirow[t]{10}{*}{ Breast } & Leuprolide & GnRH agonist: reduce estrogen levels by continuous (and not pulsate) infusion of a GnRH action mimic \\
\hline & Tamoxifen & Adjuvant hormonal treatment: blockage of estrogenic receptors (ER) in early and advanced ER-positive breast cancers \\
\hline & Exemestane & orally active aromatase ${ }^{a}$ inhibitor: irreversible blockade of estrogen production \\
\hline & Anti-aromatases & Competition with aromatase which blocks estrogen synthesis (not indicated in cited publications) \\
\hline & Doxorubicin & Antibiotic intercalating DNA agent, inhibitor of Topoisomerase II, and oxygen free radical producer leading to toxicity \\
\hline & Cyclophosphamide & Bifunctional inhibitor of DNA transcription and replication leading to mitotic cell apoptosis \\
\hline & Docetaxel & Cytotoxic properties via inhibition of the microtubule dynamic during mitosis \\
\hline & $5-\mathrm{FU}$ & Inhibition of thymidylate synthase (inhibition of DNA synthesis) \\
\hline & Vincristine & Stop tubulin polymerization and block cell during metaphase \\
\hline & Methotrexate & Inhibition of folic acid (cytotoxic effect) through inhibition of mitochondrial metabolism \\
\hline
\end{tabular}

\begin{tabular}{|c|c|c|}
\hline \multirow[t]{2}{*}{$A L L^{b}$} & Methotrexate & Inhibition of folic acid (cytotoxic effect) through inhibition of mitochondrial metabolism \\
\hline & Cytarabine & Block DNA synthesis during cell division \\
\hline \multirow[t]{4}{*}{ mRCC ${ }^{c}$ or GIST $^{d}$} & Sunitinib & Inhibition of tyrosine kinase receptors involved in tumor growth \\
\hline & Sorafenib & Kinase inhibitor which leads decrease of tumor cell proliferation \\
\hline & VEGFR inhibitors & Angiogenesis inhibitor (stop tumor growth) \\
\hline & Radiotherapy & Tumor cell apoptosis by DNA deterioration \\
\hline
\end{tabular}

${ }^{a}$ Aromatase, enzyme responsible of the biosynthesis of estrogen.

${ }^{b} A L L$, Acute lymphoblastic leukemia.

${ }^{c} m R C C$, metastatic renal cancer carcinoma.

${ }^{d}$ GIST, Gastrointestinal solid tumor.

clear data on cognition and/or biomarker levels. Of the initially selected 65 studies, 23 were finally included in the analysis (Figure 1). These studies aimed to evaluate and characterize changes in several biological factors predictive for cognitive alteration in cancer patients, often in association with treatment. Different domains of cognition were assessed by batteries of neuropsychological tests and self-reports of cognitive function. The biological factors covered in this review are summarized in Tables 2-6. Most often, blood and serum samples were analyzed as simple and rapid tests with potential to provide information about the risk of cancer patients developing cognitive issues, and to facilitate identification of optimal treatment regimens for specific patient populations.

\section{BIOLOGICAL MARKERS AND COGNITIVE IMPAIRMENTS IN TREATED CANCER PATIENTS}

\section{Plasma Biomarkers}

\section{Plasma Inflammatory Responses}

The main cytokines analyzed in the reviewed studies were the pro-inflammatory triad, interleukin-6 (IL-6), tumor necrosis factor-alpha (TNF- $\alpha$ ), and interleukin $1 \beta$ (IL-1 $\beta$ ). As IL-6 is an early mediator of inflammation and a key component of the acute phase response, it can also moderate inflammation by dampening TNF- $\alpha$ and IL- $1 \beta$ responses. Currently, the exact mechanisms involved in the inflammatory response during cancer therapy are not fully understood. Nevertheless, in cancer patients, circulating levels of cytokines were often increased and could be significant determinants of the alteration of particular cognitive functions after chemotherapy (Meyers et al., 2005; Ishikawa et al., 2012; Cheung et al., 2015). Based on the study by Ishikawa et al. (2012), it was difficult to conclusively link the observed cognitive issues with chemotherapy treatment since (i) cytokines, including IL6 , were measured in patient populations suffering from various types of advanced and inoperable or recurrent cancers and (ii) the delay between the end of the treatment and the time of the plasma assay was not stated. The study conducted by Meyers demonstrated that at baseline, higher IL-6 levels were associated with poorer executive functions, confirming that cancers are associated with high levels of circulating cytokines, connected with cognitive dysfunction, before chemotherapy. A longitudinal study by Cheung et al. (2015) established that higher concentrations of IL- $1 \beta$ and IL- 6 were associated with more severe cognitive disturbance, and that increased IL- $1 \beta$ specifically was associated with poorer response speed performance during or just after the end of a chemotherapy treatment episode (Cheung et al., 2015; Table 2). In contrast, elevated IL-4 levels were linked to better response speed and fewer cognitive complaints in patients with breast cancer (Table 2), suggesting that maintenance of IL-4 levels during cancer care is likely to be neuroprotective (Cheung et al., 2015). Interestingly, breast cancer patients treated with chemotherapy had significantly elevated IL- 6 and TNF- $\alpha$ levels after approximately 5 years offtherapy, compared with healthy controls, with an interaction between these two cytokines (Kesler et al., 2013). This study was particularly informative, since it correlated increased cytokine levels with diminished hippocampal volume, which is associated with verbal memory function. In agreement, an independent 


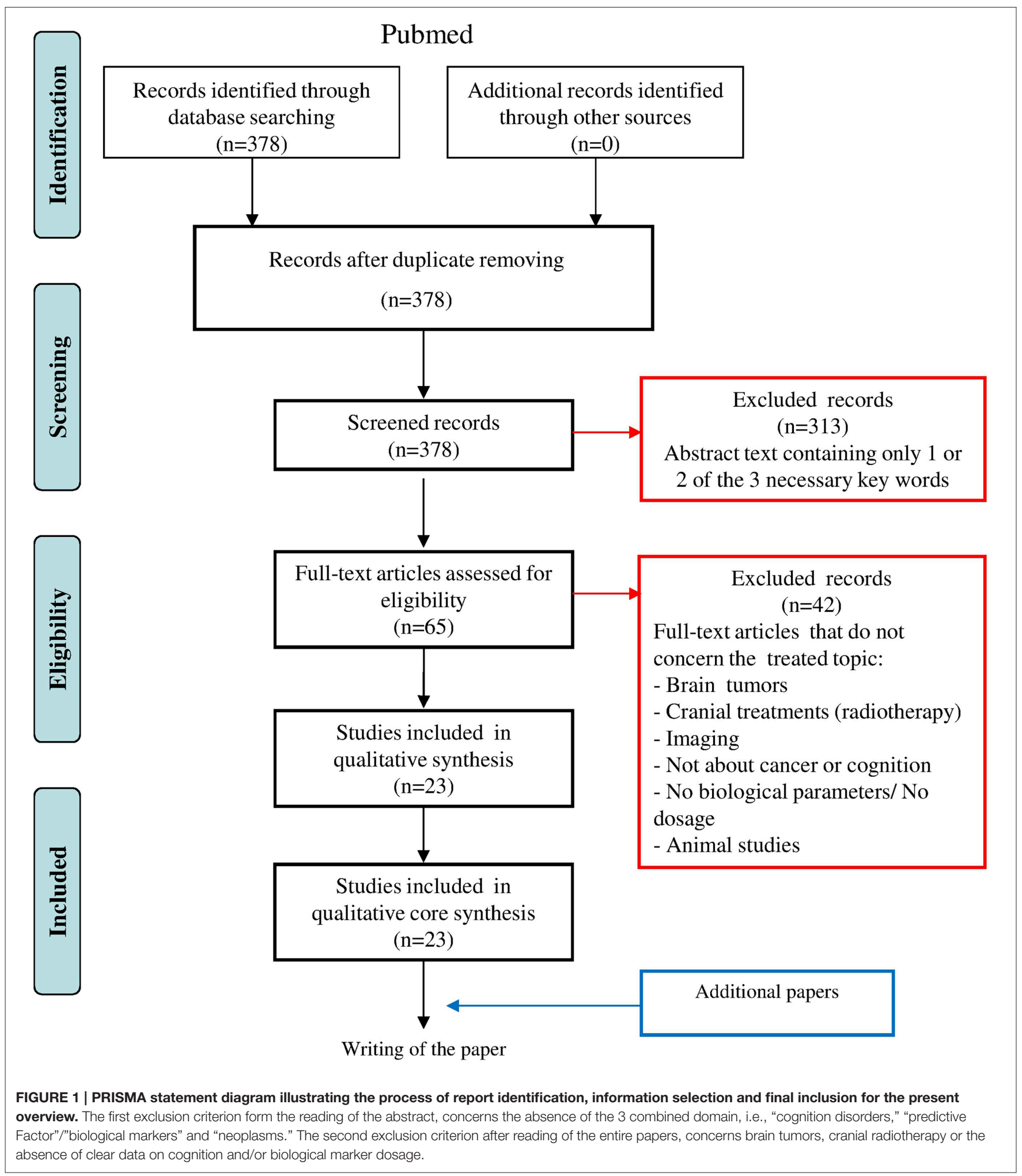

correlation between higher plasma IL-6 levels and deteriorated memory performance was described in breast cancer patients exposed to adjuvant local radiotherapy (Table 2; Shibayama et al., 2014). Together, these data suggest that cancer leads to increased plasma levels of selected pro-inflammatory cytokines, and that increased plasma IL-6 levels, likely resulting from chemotherapy or radiotherapy, may be a key systemic factor involved in, and/or predictive of, cognitive dysfunction (Table 2). 
Besides IL-6, which was described as marker of both cancer-associated and cancer treatment-induced inflammation, studies of other cytokines were less frequently reported. A longitudinal cross-sectional study, including baseline measurements, demonstrated changes in a number of proinflammatory cytokines; however, only levels of TNF receptor type-II (TNF-RII) were significantly higher in plasma from chemotherapy-treated patients compared with those who did not receive chemotherapy, with no differences observed in IL-1ra, IL-6, or C-reactive protein (CRP; Ganz et al., 2013). In detail, significant correlations between plasma TNF-RII and self-reported memory complaints, but not cognitive dysfunction evaluated by neuropsychological tests, was demonstrated at baseline (associated with relatively diminished brain metabolism). The study also demonstrated increased TNFRII over time in patients who had received radiotherapy (first end point), different chemotherapy regimens (second end point), and then endocrine therapy (third endpoint; Table 2), leading them to hypothesize that fatigue and cognitive complaints may be caused by disturbances in TNF pathways (Ganz et al., 2013).

In addition to cancer-related increases in circulating cytokine levels, data reported by Janelsins et al. (2012) support the idea that some cytokines may be specifically up-regulated in response to chemotherapy, and contribute to the development of cognitive difficulties. The study compared cytokine levels of IL-6, IL-8, and monocyte chemoattractant protein-1 (MCP-1) in patients receiving doxorubicin (Dox)based cyclophosphamide/cyclophosphamide plus fluorouracil (AC/CAF) or Dox-based cyclophosphamide, methotrexate and fluorouracil (CMF) chemotherapy. The results demonstrated augmentation and diminution of cytokine levels in the AC/CAF and CMF groups, respectively, over time from baseline (prior to chemotherapy) and after two consecutive chemotherapy cycles, with a significant difference in levels of IL- 6 between the two groups, suggesting that chemotherapy can induce specific cytokine changes. However, the study was under-powered and the time-points for blood sampling (before chemotherapy cycle) and cognitive evaluation (after chemotherapy cycle) did not match, hence it is difficult to conclusively evaluate the link between cytokine/chemokine changes and cognition, other than the reported negative correlation between MCP-1 and forgetfulness, difficulty with concentration, and thinking (subjective complaints, Table 2; Janelsins et al., 2012).

Circulating cytokines associated with cognitive impairment in cancer patients during the course of treatment, or in survivors after the end of treatment, represented the most measurable and measured factors, and studies converged to suggest that chemotherapy could dysregulate cytokine levels, which may interfere with brain functioning, leading to cognitive impairment (Ahles and Saykin, 2007). Indeed, some cytokines, including IL-6, IL-1 $\beta$, and TNF- $\alpha$, could have causal roles by crossing the BBB via active transporters (Cheung et al., 2015) and can interact with synapses (Wang et al., 2015), thereby leading to systemic communication between peripheral cytokines and the brain, rather than central cytokine production. This hypothesis is supported by a recent study by Hayslip et al. (2015), which proposed direct intravascular oxidative modification of plasma proteins by chemotherapy, leading to monocyte release of TNF- $\alpha$ which, by diffusing through the $\mathrm{BBB}$, could then activate cascades of events potentially causing cognitive impairment (Hayslip et al., 2015). They demonstrated that the exogenous anti-oxidant, sodium-2-mercaptoethane sulfonate (mesna), present only in blood and urine, reduced plasma protein oxidation and TNF$\alpha$ levels in patients receiving Dox-containing chemotherapy (Hayslip et al., 2015). Also, the observation that changes in plasma TNF- $\alpha$ levels were linked to reduced left hippocampal volume in cancer patients receiving chemotherapy (Kesler et al., 2013), suggests that cerebral apoptosis, or other cell death mechanisms, are likely responsible for altered verbal memory performance. Such cell death mechanisms could be counteracted by neutralization of circulating cytokines.

Although this hypothesis raises interesting therapeutic options, other studies did not show any significant correlation between plasma cytokine levels and cognitive impairment with or without chemotherapy (Pomykala et al., 2013). In fact, covariations between metabolism in selected brain regions and cytokines were detected by comparing values at baseline and 1 year after treatment completion in a group of patients who received chemotherapy (Pomykala et al., 2013). Overall, there is a clear difficulty in postulating a direct link between any particular cytokine that may be specifically up-regulated by a specific chemotherapy and responsible for a selected type of cognitive dysfunction. The observed discrepancies between studies may be due to differences in chemotherapy regimens, time periods between measurement of cytokine plasma levels and the end of chemotherapy, measurements of cytokine levels in serum or plasma, or the variable sensitivities of the methods used for measurement. Principal tests applied were the enzyme-linked immunosorbent assay (ELISA), including different variants such as the high sensitivity multiplex immunoassay (Ganz et al., 2013; Kesler et al., 2013; Pomykala et al., 2013; Cheung et al., 2015), or regular and high sensitivity kits (Ganz et al., 2013). Currently, the detailed mechanisms underlying cognitive changes remain unclear and future studies are required to obtain more data about the direct or indirect links between inflammatory responses and brain disorders associated with cancer therapy. It will be important to further consider the role of cytokines as predictive biomarkers for cognitive impairment in cancer and cancer-treated patients and to propose new cytokine inhibitors or antagonists as therapeutic options.

\section{Non-inflammatory Biomarkers in Blood and Serum Samples}

Since tumors can expand through development of angiogenic features and via release of angiogenetic factors, including vascular endothelial growth factor (VEGF), recently introduced targeted therapies include inhibitors of tyrosine kinase VEGF receptor (VEGFR TKI) and drugs targeting VEGF itself. As VEGF is also involved in neurogenesis and brain vascularization, it might be supposed that levels of VEGF could be linked to cognitive impairments (Table 3, Ishikawa et al., 2012). Accordingly, its levels were determined in a cross-sectional study of metastatic renal cancer (mRCC) patients treated with VEGFR TKI (8 week treatment period). The patients exhibited elevated erythrocyte 


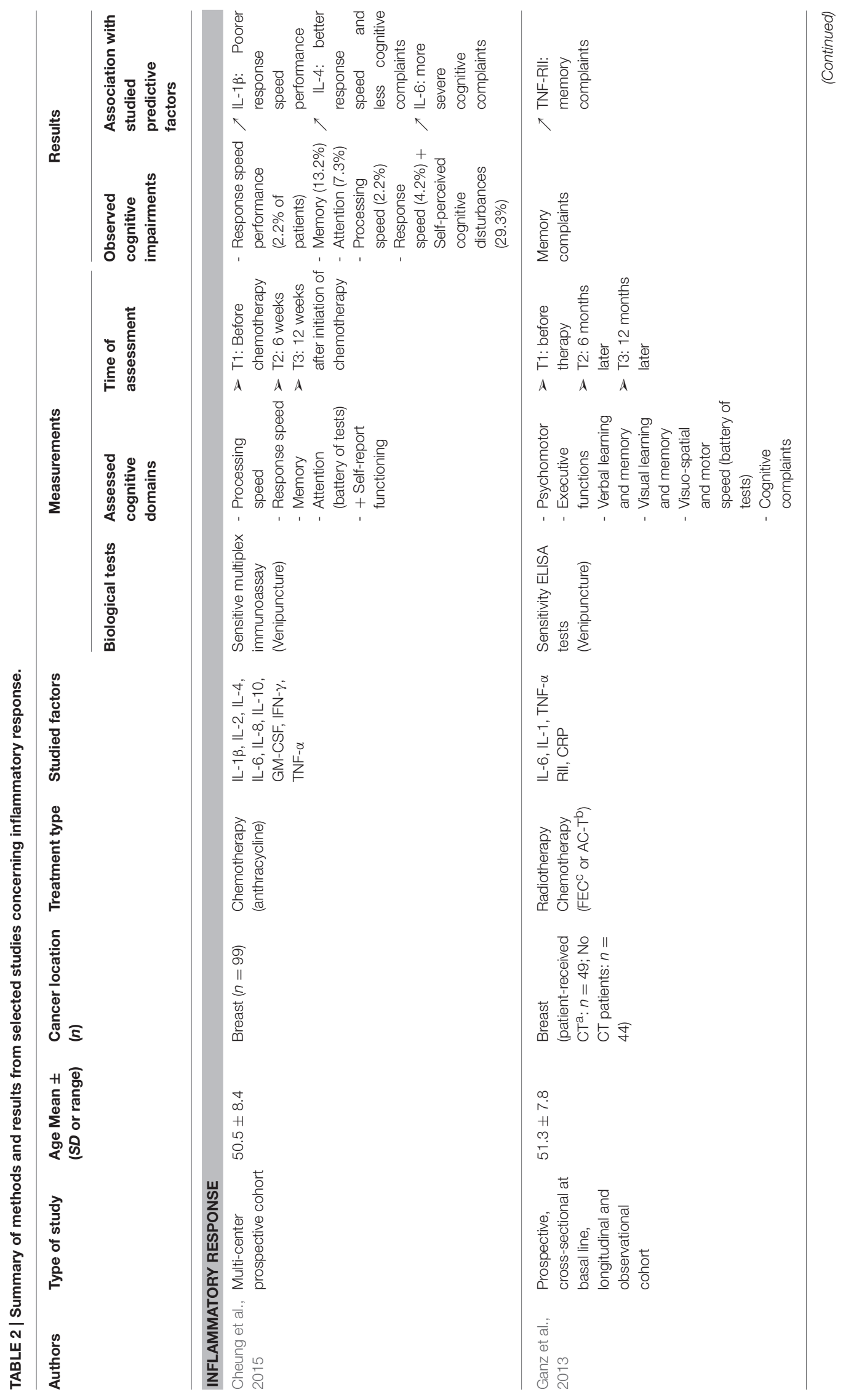




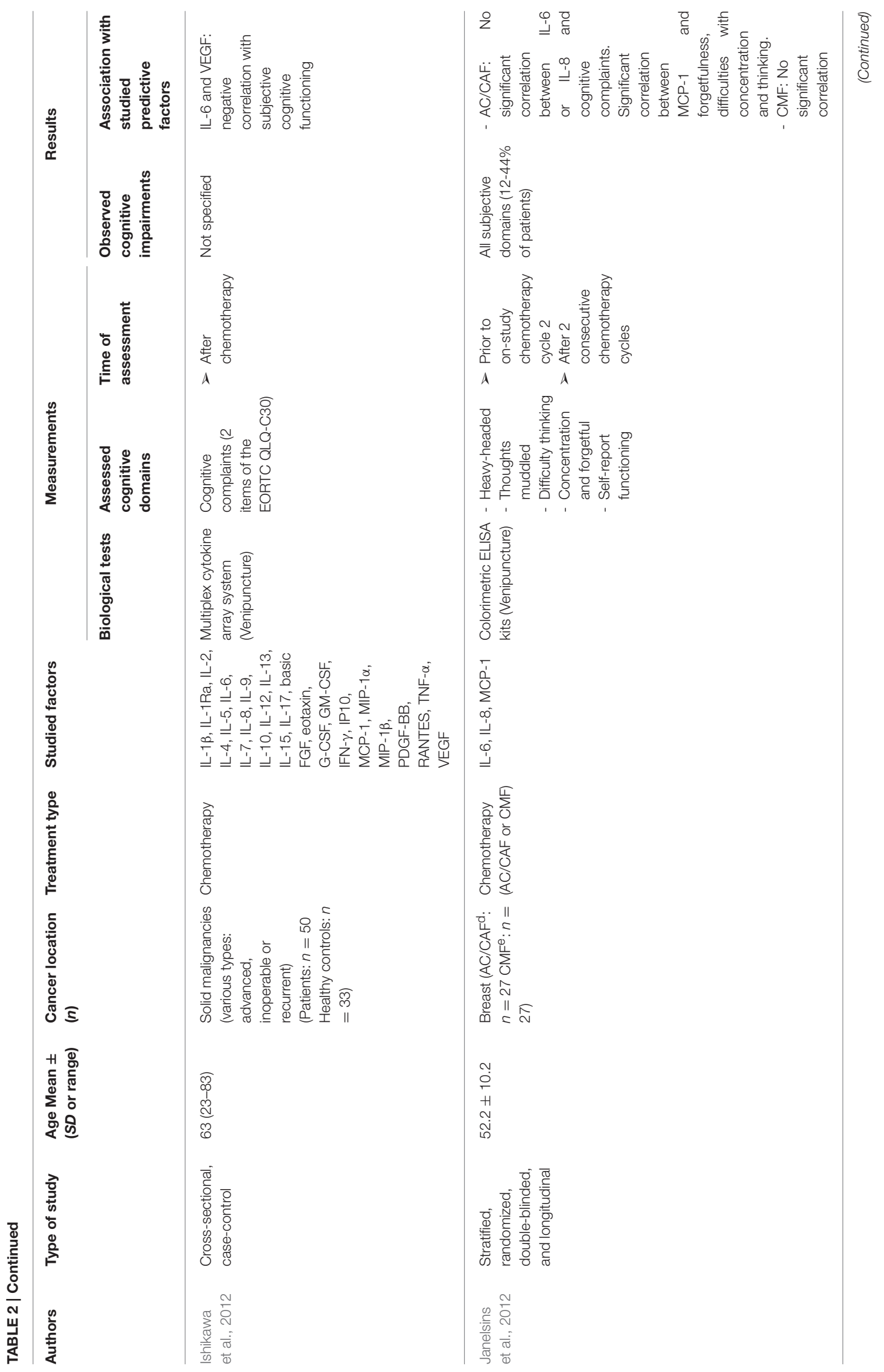




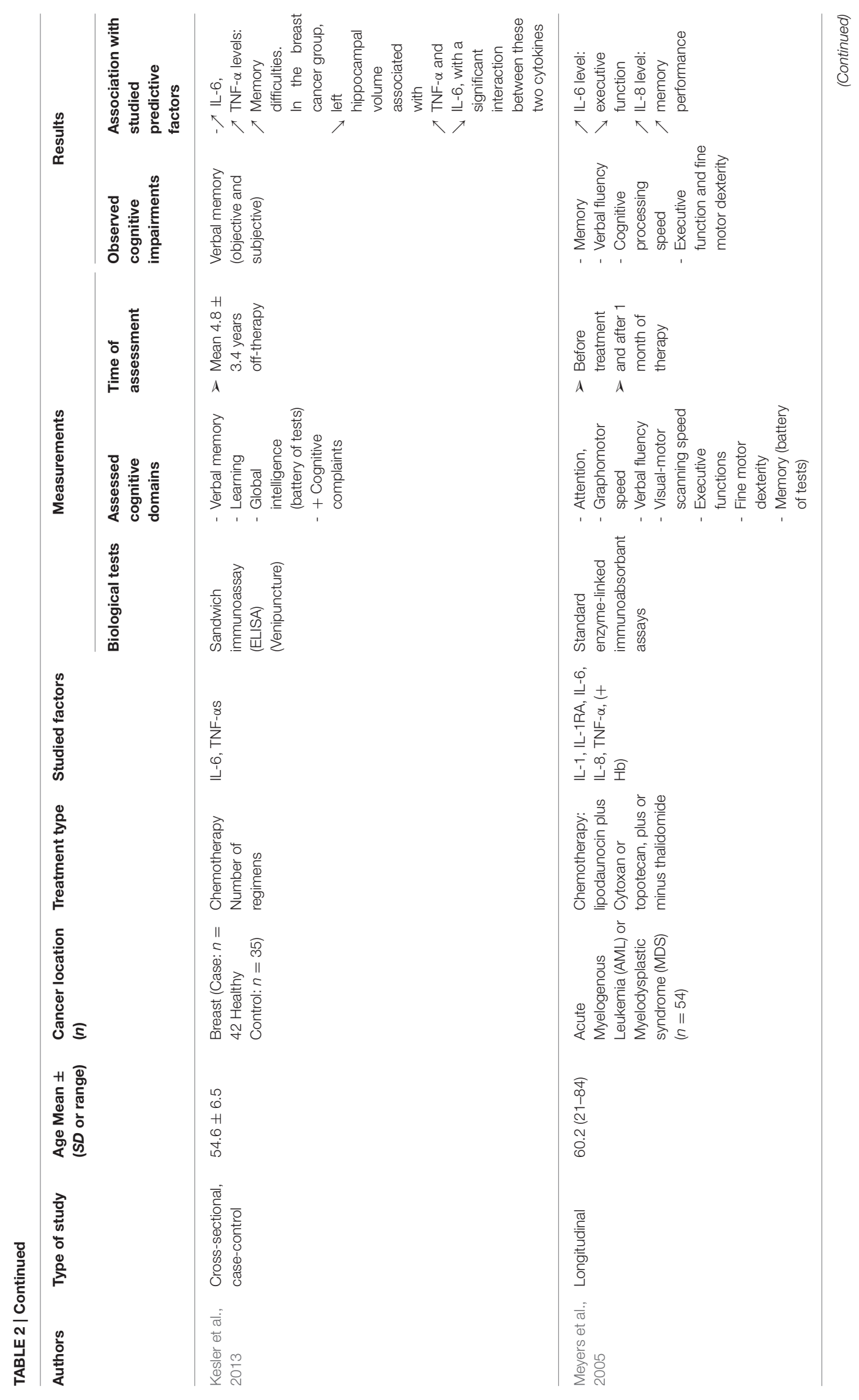




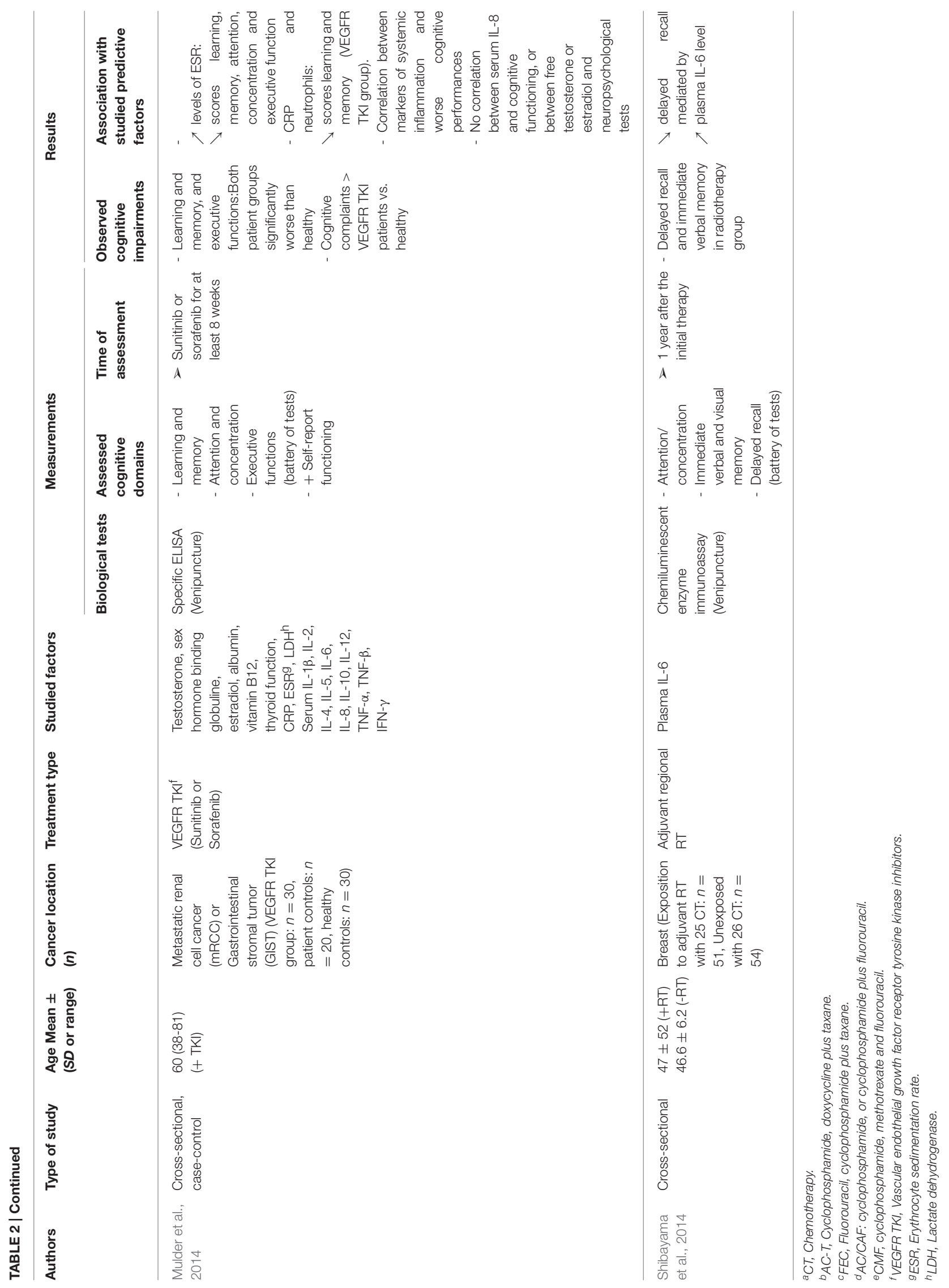




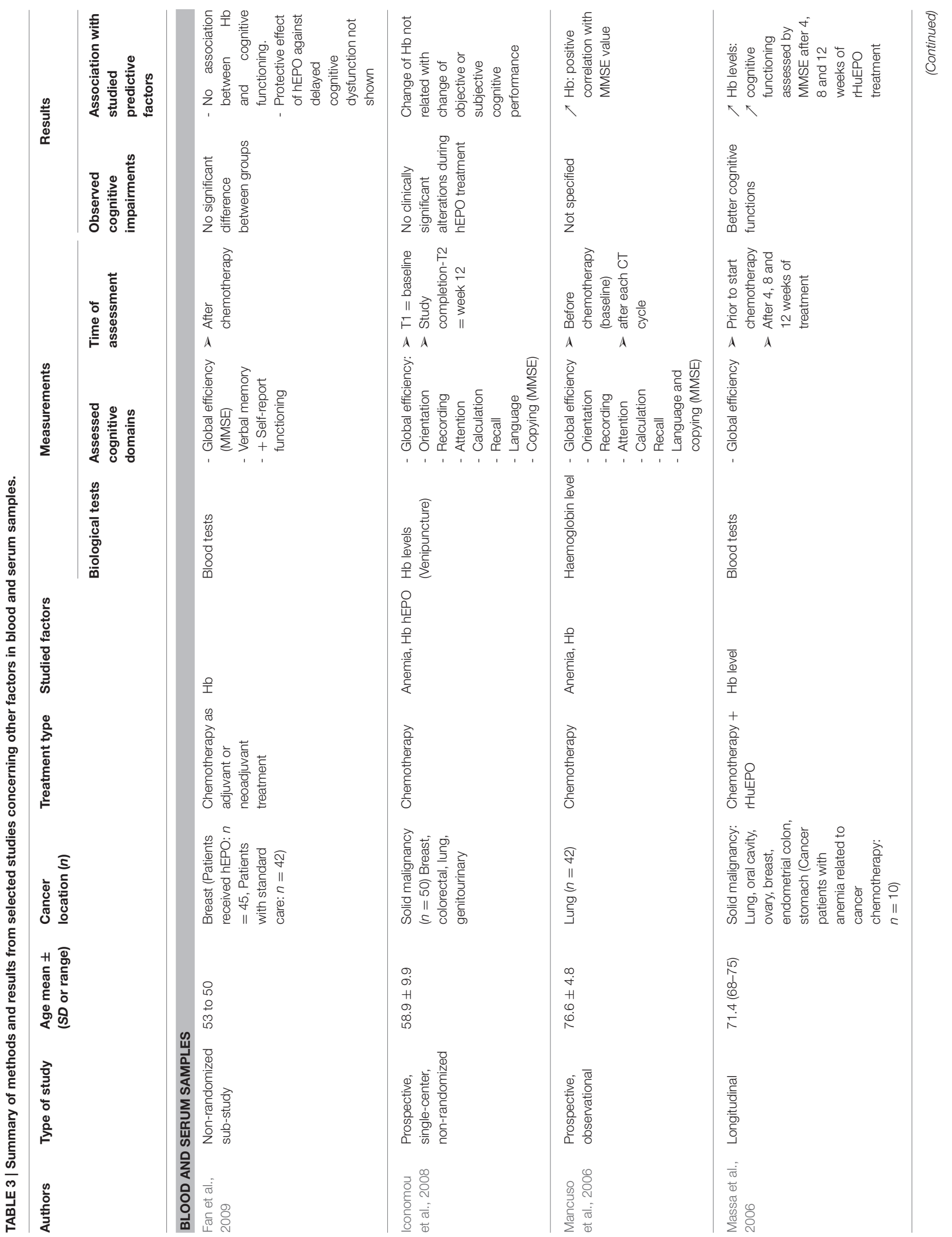




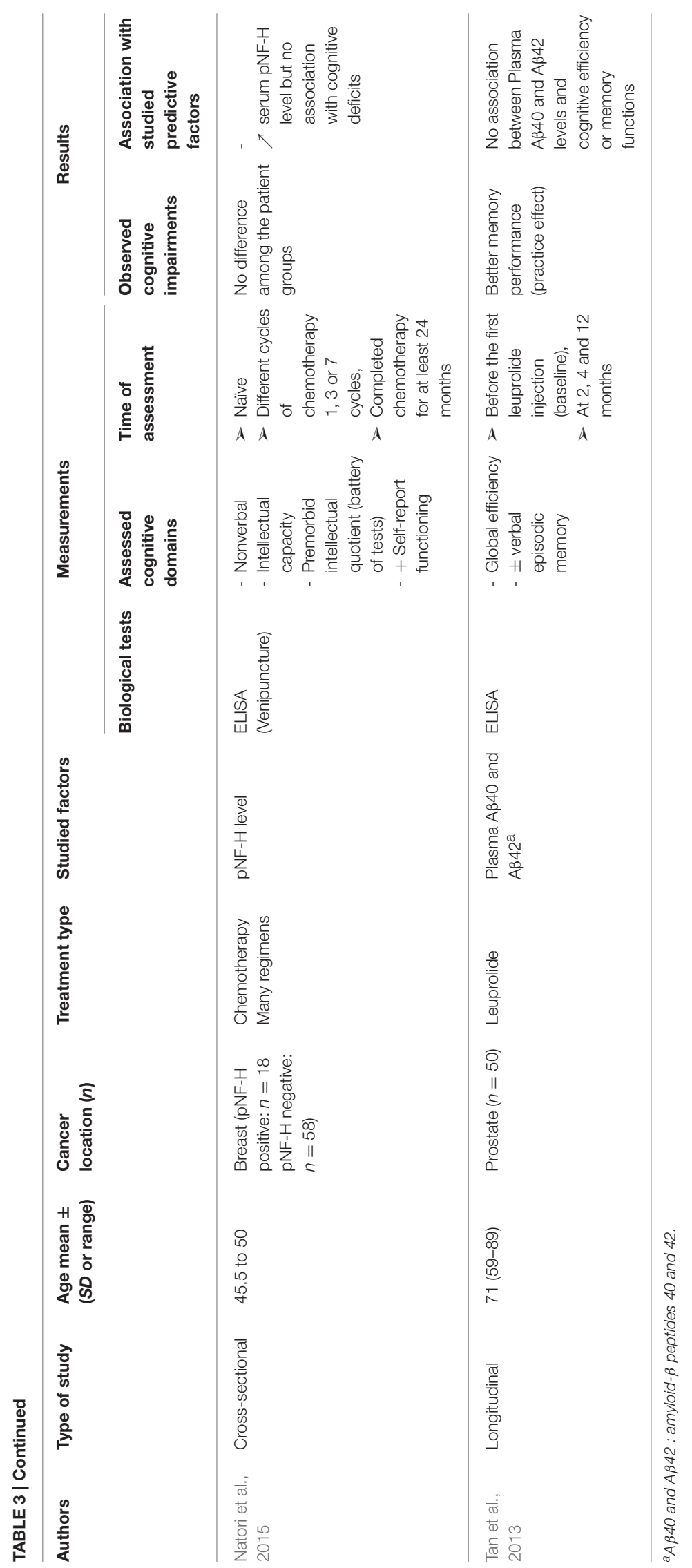




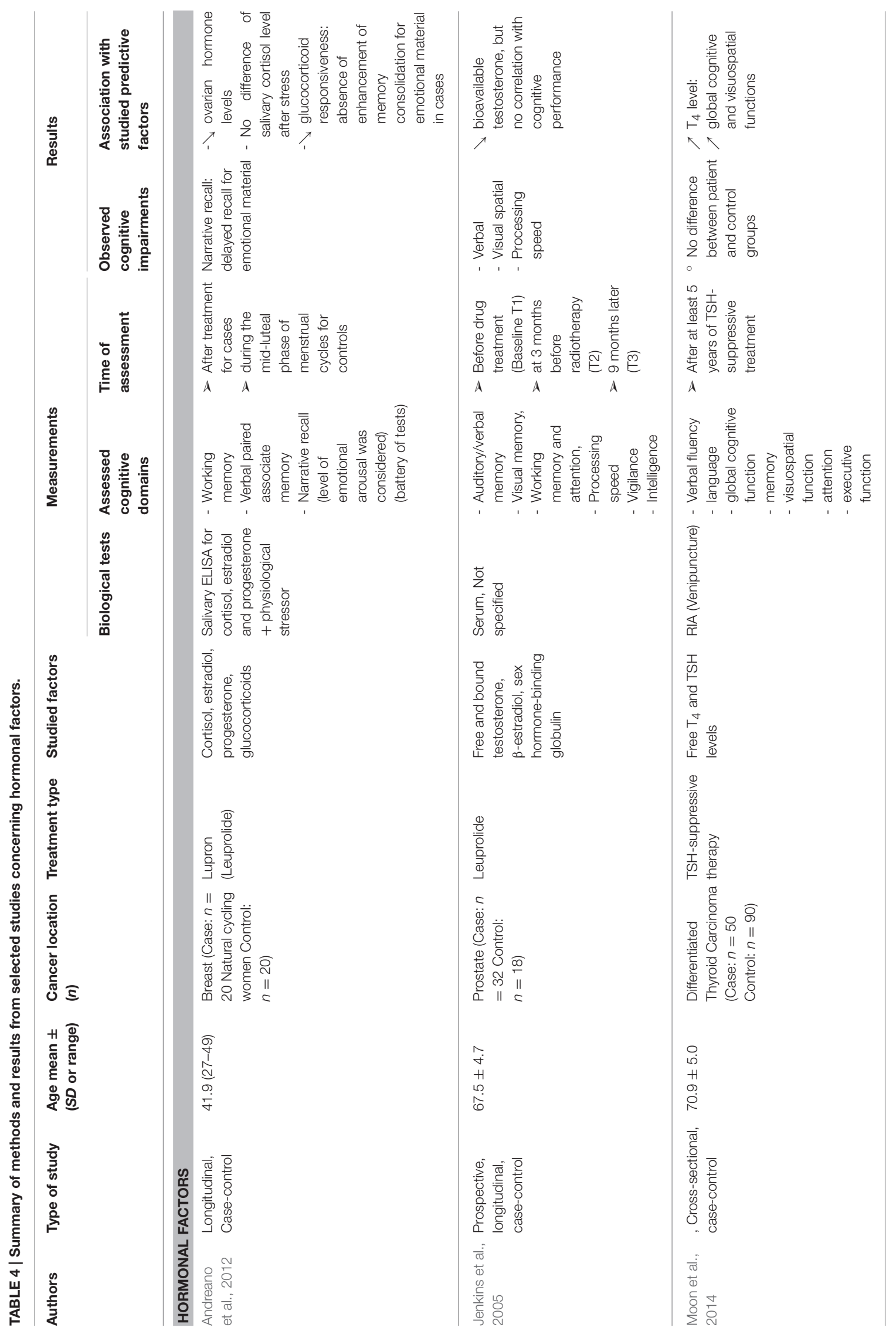




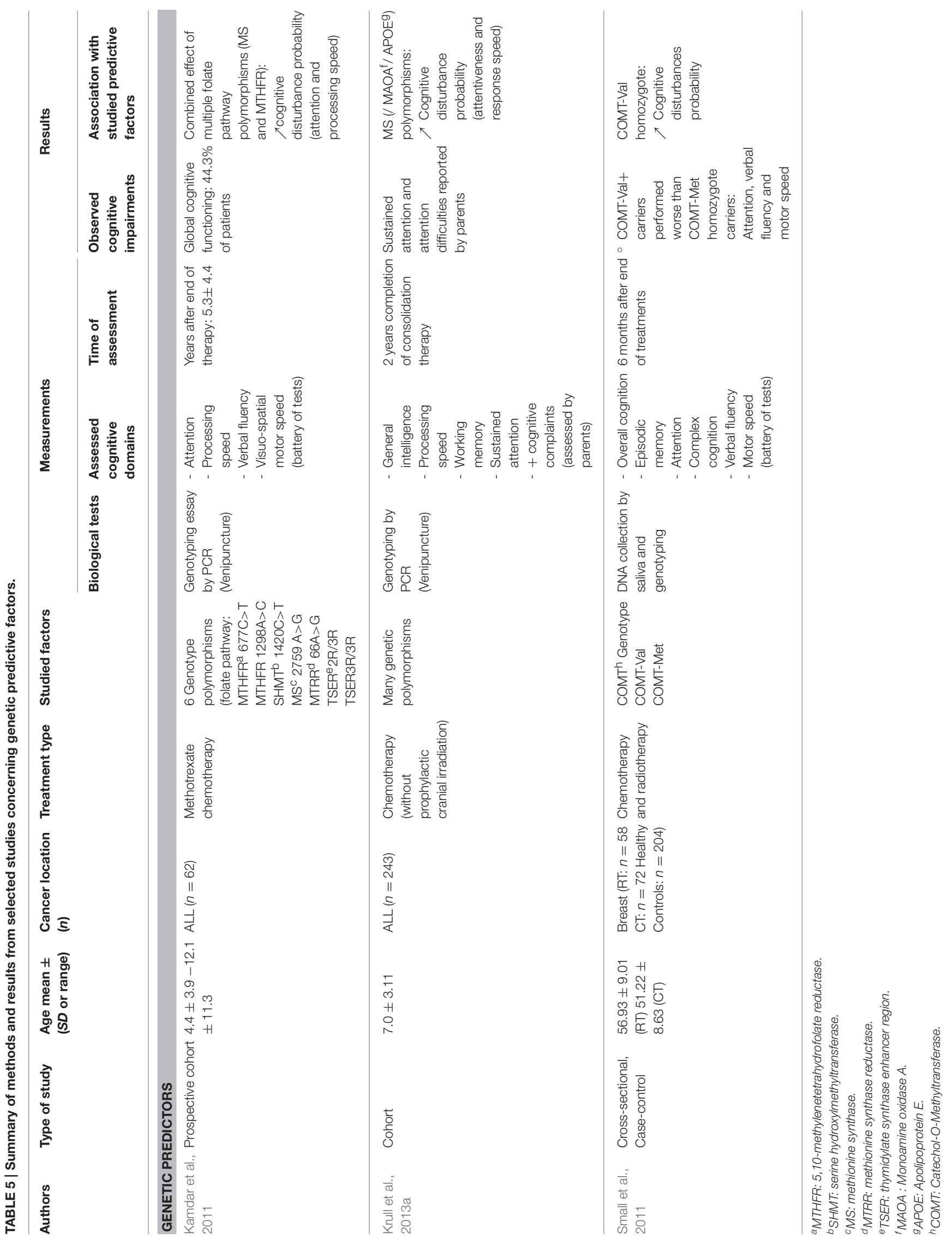




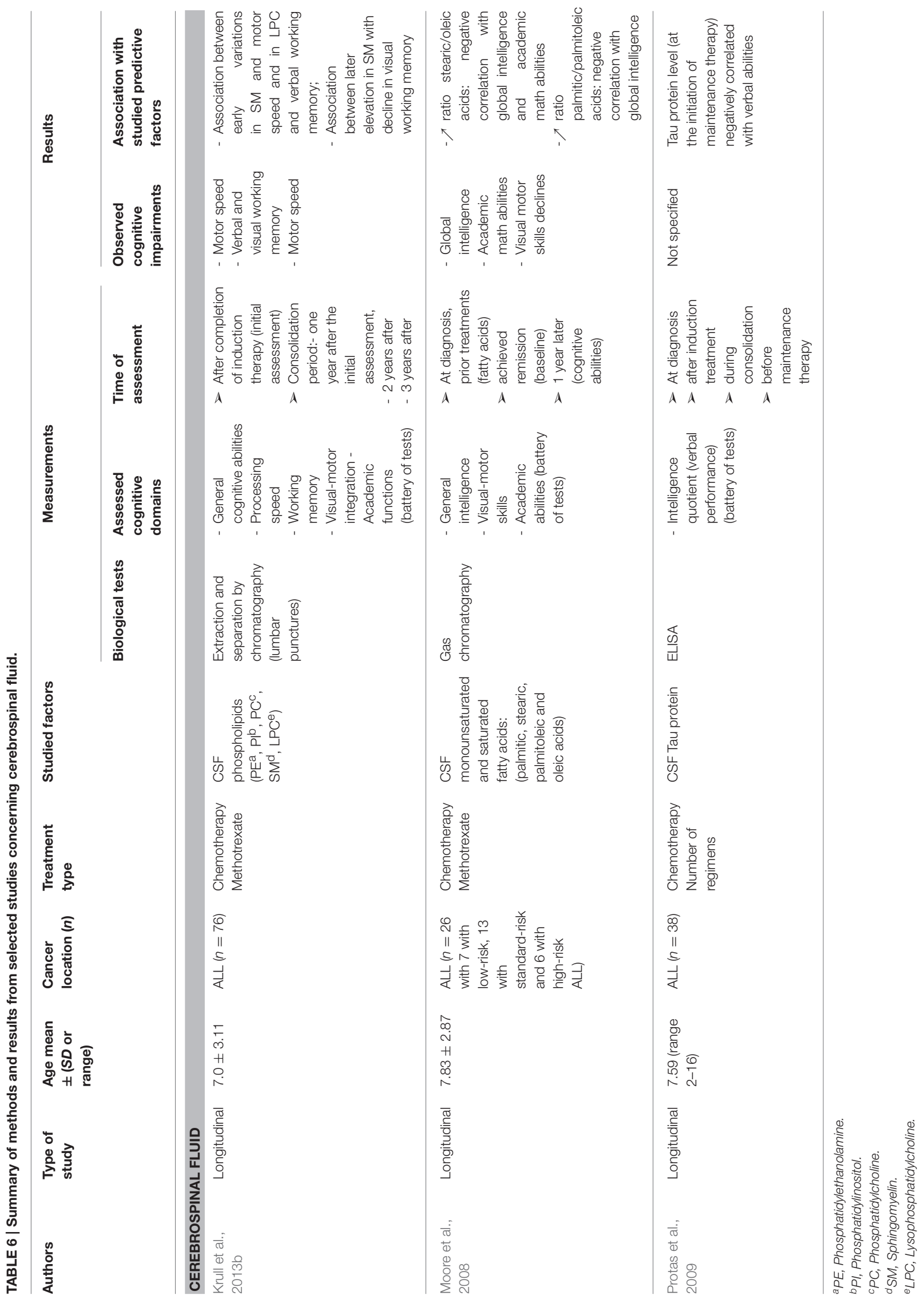


sedimentation rates (ERS), CRP levels, and neutrophil counts, that were negatively correlated with learning, memory, attention, concentration, and executive functions (Mulder et al., 2014); however, no correlations were found with cytokine, hemoglobin $\mathrm{Hb}$, or electrolyte levels, leucocyte counts, or VEGF levels in blood samples. In a more recent longitudinal study, 30\% of mRCC patients treated with anti-angiogenics were found to develop fatigue and cognitive disorders, while VEGF plasma levels measured at baseline, and 3 and 6 months from baseline, were associated with fatigue, but not with cognitive dysfunction (Joly et al., 2016). The impact of TKI, and of cancer itself, should be investigated further to clarify the exact effects of TKI on inflammatory responses and other circulating plasma markers, such as $\mathrm{Hb}$, detrimental to cognitive performance.

Several studies investigating the contribution of chemotherapy-induced anemia to cognitive impairment in cancer patients suggested that changes in $\mathrm{Hb}$ were linked to the development of cognitive impairment during chemotherapy. This was stressed in the elderly cancer population studied by Mancuso et al. (2006), were Hb levels were associated with quality of life, functional capacity, mental decline, and depression, suggesting that maintenance of normal $\mathrm{Hb}$ levels is essential to prevent cognitive decline during chemotherapy. Low powered studies on treatments with specific therapies, such as recombinant human erythropoietin (rHuEPO), led to show improved $\mathrm{Hb}$ levels which were correlated with better cognitive function (Table 3; Mancuso et al., 2006; Massa et al., 2006). When rHuEPO is administered several times each week, it can compensate for cancer and chemotherapy-induced anemia after approximately 3 weeks of chemotherapy. In a larger cohort study, Iconomou et al. (2008) observed no significant changes in cognitive function in responders, exhibiting increased $\mathrm{Hb}$ levels after 12 weeks of rHuEPO treatment, despite improvement of physical function and diminished fatigue (Iconomou et al., 2008). In contrast, other studies using the same types of cognitive tests failed to detect evidence for a protective effect of erythropoietin (EPO) against delayed cognitive dysfunction (24 months from the end of the treatment) in groups of patients with breast cancer receiving chemotherapy (Fan et al., 2009; Table 3).

Other systemic biological markers were also highlighted. A relationship between androgen receptors and amyloid precursors has been described (Takayama et al., 2009). Increased levels of amyloid- $\beta 40$ ( $\mathrm{A} \beta 40)$, a marker associated with the Alzheimer disease, did not appear to be associated with cognitive minimental state examination (MMSE) scores after leuprolide treatment in prostate cancer patients (Tan et al., 2013). However, this study had some limitations, including a lack of evaluation of the ratio $A \beta 40 / A \beta 42$ in plasma, indicating that the interesting hypothesis of a possible impact of cancer treatment on $A \beta$ plasma levels and cognition deserves further investigation (Table 3).

Another candidate plasma marker for cognitive dysfunction following therapy-induced brain damage is axonal phosphorylated neurofilament subunit $\mathrm{H}$ (pNF-H), levels of which are increased in the blood of patients who have had acute brain ischemic stroke compared with controls, and are associated with the severity of the stroke (Singh et al., 2011; Andreano et al., 2012). Thus, Natori et al. (2015) considered that pNF-H would constitute an interesting systemic biomarker of neuronal lesions and measured its levels in the serum of breast cancer patients at baseline, after one to seven cycles of different chemotherapy regimens, and 1 month-1 year after the end of therapy. They established that pNF-H levels increased with the number of chemotherapy doses administered, but did not find any correlation with neuropsychological scores (Natori et al., 2015; Table 4). This suggests that measurement of serum pNF-H in chemotherapy-treated cancer patients, alongside the application of more sensitive batteries of cognitive tests, may be worthwhile to further evaluate pNF-H as a biomarker of neural axonal damage and cognitive impairment.

\section{Hormonal Factors}

Endocrine function, specifically gonadal and stress hormones, may also contribute to cognitive difficulties during cancer treatment. To date, the results of research into hormonal factors remain inconclusive, and studies are often related to patients receiving hormonal therapy. For example, significant reductions in free testosterone and $\beta$-estradiol levels were detected in prostate cancer patients after 3 months exposure to leuprolide, and some changes in spatial memory also were observed during treatment; however, there was no association between the changes in hormonal factors and those in cognition (Jenkins et al., 2005; Table 5). When therapy to suppress thyroidstimulating hormone (TSH) was administered to patients with differentiated thyroid carcinoma, a positive correlation between free serum $\mathrm{T}_{4}$ levels and cognitive processing speed was detected (Moon et al., 2014), suggesting that exogenous $\mathrm{T}_{4}$ supplementation can improve cognitive function in this group of patients. In addition, given that gonadal hormonal levels can influence the hypothalamic-pituitary-adrenal axis (HPA), the cognitive and endocrine effects of the cortisol activating stressor, cold pressor stress (CPS), were tested in breast cancer patients previously treated with chemotherapy, and then receiving Lupron (Andreano et al., 2012). The glucocorticoid (cortisol) response to CPS was absent in the cancer patient group compared with controls, and delayed recall performance was also impaired in the individuals with cancer (Andreano et al., 2012). Thus, stress-induced cortisol favoring memory consolidation can be selectively altered in cancer patients. Other than this interesting study relating regulation of the HPA axis to cognitive impairment in cancer patients, there is little evidence to link the role of chemotherapy, stress, and/or cancer on circulating hormone levels and cognition.

\section{Genetic Factors}

There is relative heterogeneity among cancer patients regarding (i) the various domains of cognition that can be affected, including working memory, executive functions, verbal memory, and processing speed; and (ii) the proportion of patients exhibiting long-term cognitive deficits, independent of fatigue or emotional disturbances. This has prompted medical researchers to investigate potential predisposing factors for the development of cognitive impairment during cancer and its treatment. Indeed, immune status, cancer diagnosis in the elderly, and/or a number of key genetic polymorphisms can predispose to cognitive 
changes (Ahles and Saykin, 2007; Mandelblatt et al., 2013; Janelsins et al., 2014). Should studies clearly demonstrate genetic predisposition, this could enable adaption of treatment to specific patient populations. Accordingly, recent studies have shown that genetic factors may be linked to cognitive impairments in cancer patients after therapy; for example, the gene encoding apolipoprotein E (APOE), located in chromosome 19, which functions in lipid transport and regulation of inflammation. APOE has three allelic variants (E2, E3, and E4), which include various combinations of two single nucleotide polymorphisms, rs7412 and rs429358. Chemotherapy-treated breast cancer patients carrying APOE-4 allele E4, a well-known risk factor for Alzheimer's disease (Ahles, 2012), have a higher risk of cognitive dysfunction during the course of cancer treatment (Mandelblatt et al., 2013). Consistent with these findings, Krull et al. analyzed various polymorphisms among childhood ALL survivors, and identified three that were associated with neurocognitive disorders, such as attentiveness, response speed, or parent-reported attention problems. In particular, an association between APOE-4 and attention deficit was described in survivors (Table 5). This study also identified associations between a single nucleotide polymorphism in the genes encoding methionine synthase (MS), which is responsible for the conversion of homocysteine to methionine, and monoamine oxidase A (MAOA), which catalyzes the deamination of amines such as dopamine, serotonin, or norepinephrine, and attention difficulties (Krull et al., 2013a).

The key role of neurotransmitters as potential predisposing markers, is stressed by the other polymorphism commonly reported as linked to cognitive impairments, the Val158 Met encoding single-nucleotide polymorphism in catecholO-methyltransferase (COMT), which catalyzes the metabolic breakdown of catecholamines through the methylation of dopamine and noradrenaline (Ahles and Saykin, 2007). In detail, codon 158 of COMT on chromosome 22q11, can encode for either a valine or a methionine residue (Small et al., 2011). The valine-containing variant protein exhibits elevated activity, leading to enhanced neurotransmitter degradation and consequent diminished neurotransmission (Table 5). This polymorphism predisposed a subgroup of patients with breast cancer to a higher risk of diminished cognitive performance, including attention, verbal fluency, and motor speed, evaluated 6 months after the end of chemotherapy (Small et al., 2011).

Other genetic polymorphisms also appear to be implicated in cognitive changes, such as those regulating folate pathways. Kamdar et al. (2011) investigated six different polymorphisms in genes involved in the folate pathway in childhood ALL survivors (Kamdar et al., 2011; Table 5), and described two genotypes in genes encoding 10-methylenetetrahydrofolate reductase and sphingomyelin (SM) as significantly correlated with general neurocognitive impairment (Kamdar et al., 2011).

\section{Biological Factors in Cerebrospinal Fluid}

When attempting to identify direct biological factors associated with cognitive alterations in cancer patients, variations in levels detected in cerebrospinal fluid (CSF) would be expected to provide better information about causal links with, or consequences of, treatment. Relationships between alterations in phospholipids, SM, and lysophosphatidylcholine (LPC) concentrations, as markers of white matter integrity, and some domains of cognitive function, were identified in children with ALL before and during long periods of chemotherapy (methotrexate administration over a period of years; Krull et al., 2013b). SM and LPC were shown to increase in CSF following chemotherapy induction and were associated with motor speed or visual working memory, and verbal working memory, respectively (Table 6; Krull et al., 2013b). These data indicate the occurrence of early cerebral neurochemical and neurocognitive alterations during chemotherapy, suggesting that, in addition to the effects of cancer itself, there is a direct and rapid impact of chemotherapy on the brain (white matter), and that brain imaging of the white matter may be beneficial during methotrexate administration. Methotrexate alters mitochondrial oxidative metabolism by inhibiting recycling of nicotinamide adenine dinucleotide, leading to accumulation of monounsaturated fatty acids. Thus, Moore et al. (2008) evaluated fatty acid levels (ratio between monounsaturated/saturated) in the CSF of patients with childhood ALL treated with methotrexate for more than 3 years (Moore et al., 2008). The number of intrathecal methotrexate doses received during the first year was significantly correlated with an increase in the stearic/oleic acid ratio, which was negatively correlated with decreased global intelligence and academic mathematics ability, while the palmitic/palmitoleic acid ratio was negatively correlated with global intelligence alone (Table 6; Moore et al., 2008). Hence, these two studies strongly support a specific deleterious impact of chemotherapy on beta-oxidation and fatty acid metabolism in the brain, suggesting that membrane and myelin defects may accompany cognitive dysfunction in some populations of cancer patients.

CSF analysis can also provide inform about the microtubuleassociated protein tau, whose CSF levels have already been associated with neurotoxicity and neurodegenerative pathologies. There is a significant increase in tau protein after induction and during consolidation, compared with at the time of diagnosis, in ALL patients. The level of tau measured before maintenance therapy was negatively correlated with verbal abilities (Protas et al., 2009), suggesting probable neural cell injury.

Overall, studies of patients with ALL receiving methotrexatecontaining chemotherapy regimens for long periods demonstrate robust links between cognitive domains, such as working memory or verbal abilities, and modified CSF components, such as fatty acids, phospholipids, and even tau protein, which plays an important role in Alzheimer's disease (Table 6). Although the sampling method to obtain CSF by lumbar puncture is more invasive than blood tests, it appears to provide promising predictive biological information relating to cognitive function in cancer patients, which could be highly useful in various settings.

\section{Co-Morbidities and Limitations}

It is important to consider clinical, physiopathological, and psychological factors in addition to biological markers, in relation to cognitive impairment of patients with cancer. In 
particular, to evaluate the contribution of co-morbidities and associated treatments, is essential to understand patient history and knowledge of these factors can help to predict cognitive impairments and determine the significance of changes in circulating factors in cancer patients during treatment. In support of this idea, in a study aiming to identify predictors of cognitive performance in breast cancer patients, treatment for hypertension was identified as having a significant negative impact on verbal fluency and working memory performance, and treatment for diabetes mellitus, was found to detrimentally affect executive functioning and reaction speed (Schilder et al., 2010). The same study also demonstrated that a higher number of "reproductive years" (as an indicator of lifetime estrogen exposure) appears to predict worse executive functioning. A longitudinal cohort study by Bender et al. (2013) demonstrated that before adjuvant chemotherapy, post-menopausal breast cancer patients exhibit poorer cognitive function than matched healthy controls; however, factors related to oral contraception were better predictors of verbal memory and attention in both controls and cancer patients (Bender et al., 2013), likely due to the positive biological impact of estrogen on brain function. The roles of various other factors, such as surgery, sleep disorders, anxiety, and cancer itself, on cognitive impairments specifically observed in cancer patients before chemotherapy, remain unknown. More generally, a study by Mandelblatt et al. (2014) revealed that elderly breast cancer patients with more advanced cancer or high levels of co-morbidity (including diabetes and cardiovascular disease) had higher rates of cognitive impairment than those with low co-morbidity levels, unlike matched control groups (Mandelblatt et al., 2014). These results highlight that some cancer patient populations are at risk of developing cognitive deficits as a result of cancer management, including chemotherapy.

Several limitations should be noted in the studies analyzed in this report. There is an absence of meta-analyses, and the majority of available studies were prospective cross-sectional trials, mostly composed of small samples, and consequently had relatively low statistical power. Also, the studies included are not strictly comparable, because of the different methods used. Biological measurement methods are the main limit, and thus the variability in assessed cognitive domains and tests analyzed should also be considered in evaluation of this review. Indeed, some studies use global efficiency analyses, such as MMSE, whereas others applied batteries of tests, which are more sensitive for objective measurement of cognitive impairments. It should also be noted that practice effects can modify test results, particularly in longitudinal studies repeatedly using the same tests on patients after short periods of time. Finally, the large diversity of chemotherapy regimens used, inconsistent sampling points, and various cognitive assessment methods remain the

\section{REFERENCES}

Ahles, T. A. (2012). Brain vulnerability to chemotherapy toxicities. Psychooncology 21, 1141-1148. doi: 10.1002/pon.3196 major obstacles to identification of clear correlations between circulating biological factors levels and performance in specific domains of cognition. In addition, brain imaging could be an interesting approach to correlation of brain activity and biological markers in patients exhibiting no obvious cognitive impairment (Ferguson et al., 2007).

\section{CONCLUSION}

A number of potential predictive markers have been identified that require validation in large series. Indeed, initial studies of factors, such as selected cytokines, stress hormones, CSF proteins, lipids, or $\mathrm{Hb}$ levels, have provided interesting information about changes in biomarkers that evolve during the course of the treatment of cancer patients, and also about genetic polymorphisms predisposing to cognitive deficits. Additional longitudinal studies, and investigation of other factors, previously identified in different pathological situations as associated with fatigue or aging, should facilitate better characterization of risk of cognitive impairment in cancer.

The question addressed in this study is among the priorities in cancer patient care and the ability to use biological risk factors to predict, better understand, and help to prevent cognitive issues, or adjust treatments for specific populations of patients identified as at risk, would be of major benefit. Such markers would also likely facilitate identification of biological mechanisms underlying neurotoxicity, and could open new avenues for testing and evaluation of therapeutic strategies designed to prevent cognitive dysfunction during cancer treatment, leading to improved quality of life, autonomy, and return to work rates of cancer survivors.

\section{AUTHOR CONTRIBUTIONS}

$\mathrm{HC}$ and $\mathrm{AD}$ selected, read and analyzed articles. ML, MT, and MD built tables and analyzed cognitive domains evaluated in each study. HC and FJ supervised, organized and wrote the manuscript.

\section{FUNDING}

Academic French governemental organization: Inserm and Normandie University, Anti-cancer center: Baclesse center, Caen, France

\section{ACKNOWLEDGMENTS}

We thank Inserm, the Baclesse Center, the Normandie Rouen and Caen Université, and the Cancéropôle Nord-Ouest that aided the efforts of the authors. 
consequences of acute stress. Psychooncology 21, 1091-1098. doi: 10.1002/ pon.2006

Bender, C. M., Sereika, S. M., Ryan, C. M., Brufsky, A. M., Puhalla, S., and Berga, S. L. (2013). Does lifetime exposure to hormones predict pretreatment cognitive function in women before adjuvant therapy for breast cancer? Menopause 20, 922-929. doi: 10.1097/GME.0b013e3182 $843 \mathrm{eff}$

Cheung, Y. T., Ng, T., Shwe, M., Ho, H. K., Foo, K. M., Cham, M. T., et al. (2015). Association of proinflammatory cytokines and chemotherapyassociated cognitive impairment in breast cancer patients: a multi-centered, prospective, cohort study. Ann. Oncol. 26, 1446-1451. doi: 10.1093/annonc/ mdv206

Fan, H. G. M., Park, A., Xu, W., Yi, Q.-L., Braganza, S., Chang, J., et al. (2009). The influence of erythropoietin on cognitive function in women following chemotherapy for breast cancer. Psychooncology 18, 156-161. doi: $10.1002 /$ pon. 1372

Ferguson, R. J., McDonald, B. C., Saykin, A. J., and Ahles, T. A. (2007). Brain structure and function differences in monozygotic twins: possible effects of breast cancer chemotherapy. J. Clin. Oncol. 25, 3866-3870. doi: 10.1200/JCO.2007.10.8639

Ganz, P. A., Bower, J. E., Kwan, L., Castellon, S. A., Silverman, D. H. S., Geist, C., et al. (2013). Does tumor necrosis factor-alpha $(\mathrm{TNF}-\alpha)$ play a role in post-chemotherapy cerebral dysfunction? Brain. Behav. Immun. 30(Suppl.), S99-S108. doi: 10.1016/j.bbi.2012. 07.015

Hayslip, J., Dressler, E. V., Weiss, H., Taylor, T. J., Chambers, M., Noel, T., et al. (2015). Plasma TNF- $\alpha$ and soluble TNF receptor levels after doxorubicin with or without Co-Administration of Mesna-A randomized, cross-over clinical study. PLoS ONE 10:e0124988. doi: 10.1371/journal.pone. 0124988

Iconomou, G., Koutras, A., Karaivazoglou, K., Kalliolias, G. D., Assimakopoulos, K., Argyriou, A. A., et al. (2008). Effect of epoetin alpha therapy on cognitive function in anaemic patients with solid tumours undergoing chemotherapy. Eur. J. Cancer Care (Engl) 17, 535-541. doi: 10.1111/j.1365-2354.2007. 00857.x

Ishikawa, T., Kokura, S., Sakamoto, N., Okajima, M., Matsuyama, T., Sakai, H., et al. (2012). Relationship between circulating cytokine levels and physical or psychological functioning in patients with advanced cancer. Clin. Biochem. 45, 207-211. doi: 10.1016/j.clinbiochem.2011. 09.007

Janelsins, M. C., Kesler, S. R., Ahles, T. A., and Morrow, G. R. (2014). Prevalence, mechanisms, and management of cancer-related cognitive impairment. Int. Rev. Psychiatry 26, 102-113. doi: 10.3109/09540261.2013. 864260

Janelsins, M. C., Mustian, K. M., Palesh, O. G., Mohile, S. G., Peppone, L. J., Sprod, L. K., et al. (2012). Differential expression of cytokines in breast cancer patients receiving different chemotherapies: implications for cognitive impairment research. Support. Care Cancer 20, 831-839. doi: 10.1007/s00520-011-1158-0

Jenkins, V. A., Bloomfield, D. J., Shilling, V. M., and Edginton, T. L. (2005). Does neoadjuvant hormone therapy for early prostate cancer affect cognition? Results from a pilot study. BJU Int. 96, 48-53. doi: 10.1111/j.1464-410X.2005.05565.X

Joly, F., Giffard, B., Rigal, O., De Ruiter, M. B., Small, B. J., Dubois, M., et al. (2015). Impact of cancer and its treatments on cognitive function: advances in research from the Paris International Cognition and Cancer Task Force Symposium and update since 2012. J. Pain Symptom Manag. 50, 830-841. doi: 10.1016/j.jpainsymman.2015.06.019

Joly, F., Heutte, N., Duclos, B., Noal, S., Léger, I., Dauchy, S., et al. (2016). Prospective evaluation of the impact of antiangiogenic treatment on fatigue and cognitive functions in metastatic renal cancer. Eur. Urol. Focus. doi: 10.1016/j. euf.2016.04.009

Kamdar, K. Y., Krull, K. R., El-Zein, R. A., Brouwers, P., Potter, B. S., Harris, L. L., et al. (2011). Folate pathway polymorphisms predict deficits in attention and processing speed after childhood leukemia therapy. Pediatr. Blood Cancer 57, 454-460. doi: 10.1002/pbc.23162

Kesler, S., Janelsins, M., Koovakkattu, D., Palesh, O., Mustian, K., Morrow, G., et al. (2013). Reduced hippocampal volume and verbal memory performance associated with interleukin-6 and tumor necrosis factor-alpha levels in chemotherapy-treated breast cancer survivors. Brain. Behav. Immun. 30(Suppl.), S109-S116. doi: 10.1016/j.bbi.2012.05.017

Krull, K. R., Bhojwani, D., Conklin, H. M., Pei, D., Cheng, C., Reddick, W. E., et al. (2013a). Genetic mediators of neurocognitive outcomes in survivors of childhood acute lymphoblastic leukemia. J. Clin. Oncol. 31, 2182-2188. doi: 10.1200/JCO.2012.46.7944

Krull, K. R., Hockenberry, M. J., Miketova, P., Carey, M., and Moore, I. M. (2013b). Chemotherapy-related changes in central nervous system phospholipids and neurocognitive function in childhood acute lymphoblastic leukemia. Leuk. Lymphoma 54, 535-540. doi: 10.3109/10428194.2012. 717080

Mancuso, A., Migliorino, M., De Santis, S., Saponiero, A., and De Marinis, F. (2006). Correlation between anemia and functional/cognitive capacity in elderly lung cancer patients treated with chemotherapy. Ann. Oncol. 17, 146-150. doi: 10.1093/annonc/mdj038

Mandelblatt, J. S., Hurria, A., McDonald, B. C., Saykin, A. J., Stern, R. A., VanMeter, J. W., et al. (2013). Cognitive effects of cancer and its treatments at the intersection of aging: what do we know; what do we need to know? Semin. Oncol. 40, 709-725. doi: 10.1053/j.seminoncol.2013.09.006

Mandelblatt, J. S., Stern, R. A., Luta, G., McGuckin, M., Clapp, J. D., Hurria, A., et al. (2014). Cognitive impairment in older patients with breast cancer before systemic therapy: is there an interaction between cancer and comorbidity? J. Clin. Oncol. 32, 1909-1918. doi: 10.1200/JCO.2013. 54.2050

Massa, E., Madeddu, C., Lusso, M. R., Gramignano, G., and Mantovani, G. (2006). Evaluation of the effectiveness of treatment with erythropoietin on anemia, cognitive functioning and functions studied by comprehensive geriatric assessment in elderly cancer patients with anemia related to cancer chemotherapy. Crit. Rev. Oncol. Hematol. 57, 175-182. doi: 10.1016/j.critrevonc.2005.06.001

Meyers, C. A., Albitar, M., and Estey, E. (2005). Cognitive impairment, fatigue, and cytokine levels in patients with acute myelogenous leukemia or myelodysplastic syndrome. Cancer 104, 788-793. doi: 10.1002/cncr.21234

Moon, J. H., Ahn, S., Seo, J., Han, J. W., Kim, K. M., Choi, S. H., et al. (2014). The effect of long-term thyroid-stimulating hormone suppressive therapy on the cognitive function of elderly patients with differentiated thyroid carcinoma. J. Clin. Endocrinol. Metab. 99, 3782-3789. doi: 10.1210/jc. 2013-4454

Moore, I. M. K., Miketova, P., Hockenberry, M., Krull, K., Pasvogel, A., Carey, M., et al. (2008). Methotrexate-induced alterations in beta-oxidation correlate with cognitive abilities in children with acute lymphoblastic leukemia. Biol. Res. Nurs. 9, 311-319. doi: 10.1177/1099800407313268

Mulder, S. F., Bertens, D., Desar, I. M. E., Vissers, K. C. P., Mulders, P. F. A., Punt, C. J. A., et al. (2014). Impairment of cognitive functioning during Sunitinib or Sorafenib treatment in cancer patients: a cross sectional study. BMC Cancer 14:219. doi: 10.1186/1471-2407-14-219

Natori, A., Ogata, T., Sumitani, M., Kogure, T., Yamauchi, T., and Yamauchi, H. (2015). Potential role of pNF-H, a biomarker of axonal damage in the central nervous system, as a predictive marker of chemotherapyinduced cognitive impairment. Clin. Cancer Res. 21, 1348-1352. doi: 10.1158/1078-0432.CCR-14-2775

Pomykala, K. L., Ganz, P. A., Bower, J. E., Kwan, L., Castellon, S. A., Mallam, S., et al. (2013). The association between pro-inflammatory cytokines, regional cerebral metabolism, and cognitive complaints following adjuvant chemotherapy for breast cancer. Brain Imaging Behav. 7, 511-523. doi: 10.1007/s11682-013-9243-2

Protas, P. T., Muszynska-Roslan, K., Holownia, A., Grabowska, A., Wielgat, P., Krawczuk-Rybak, M., et al. (2009). Negative correlation between cerebrospinal fluid tau protein and cognitive functioning in children with acute lymphoblastic leukemia. Pediatr. Blood Cancer 53, 105-108. doi: 10.1002/pbc. 900022029

Schilder, C. M. T., Seynaeve, C., Linn, S. C., Boogerd, W., Beex, L. V., Gundy, C. M., et al. (2010). Cognitive functioning of postmenopausal breast cancer patients before adjuvant systemic therapy, and its association with medical and psychological factors. Crit. Rev. Oncol. Hematol. 76, 133-141. doi: 10.1016/j.critrevonc.2009.11.001

Shibayama, O., Yoshiuchi, K., Inagaki, M., Matsuoka, Y., Yoshikawa, E., Sugawara, Y., et al. (2014). Association between adjuvant regional 
radiotherapy and cognitive function in breast cancer patients treated with conservation therapy. Cancer Med. 3, 702-709. doi: 10.1002/ cam 4.174

Singh, P., Yan, J., Hull, R., Read, S., O'Sullivan, J., Henderson, R. D., et al. (2011). Levels of phosphorylated axonal neurofilament subunit $\mathrm{H}(\mathrm{pNfH})$ are increased in acute ischemic stroke. J. Neurol. Sci. 304, 117-121. doi: 10.1016/j.jns.2011. 01.025

Small, B. J., Rawson, K. S., Walsh, E., Jim, H. S. L., Hughes, T. F., Iser, L., et al. (2011). Catechol-O-methyltransferase genotype modulates cancer treatmentrelated cognitive deficits in breast cancer survivors. Cancer 117, 1369-1376. doi: $10.1002 /$ cncr. 25685

Takayama, K., Tsutsumi, S., Suzuki, T., Horie-Inoue, K., Ikeda, K., Kaneshiro, K., et al. (2009). Amyloid precursor protein is a primary androgen target gene that promotes prostate cancer growth. Cancer Res. 69, 137-142. doi: 10.1158/0008-5472.CAN-08-3633

Tan, W. W., Heckman, M. G., Vishnu, P., Crook, J. E., Younkin, L. H., Covil, E. G., et al. (2013). Effect of leuprolide on serum amyloid$\beta$ peptide levels and memory in patients with prostate cancer with biochemical recurrence. Urology 81, 150-154. doi: 10.1016/j.urology.2012.0 8.066
Vardy, J., Wefel, J. S., Ahles, T., Tannock, I. F., and Schagen, S. B. (2008). Cancer and cancer-therapy related cognitive dysfunction: an international perspective from the Venice cognitive workshop. Ann. Oncol. 19, 623-629. doi: 10.1093/annonc/mdm500

Wang, X.-M., Walitt, B., Saligan, L., Tiwari, A. F. Y., Cheung, C. W., and Zhang, Z.-J. (2015). Chemobrain: a critical review and causal hypothesis of link between cytokines and epigenetic reprogramming associated with chemotherapy. Cytokine 72, 86-96. doi: 10.1016/j.cyto.2014.12.006

Conflict of Interest Statement: The authors declare that the research was conducted in the absence of any commercial or financial relationships that could be construed as a potential conflict of interest.

Copyright $\odot 2017$ Castel, Denouel, Lange, Tonon, Dubois and Joly. This is an openaccess article distributed under the terms of the Creative Commons Attribution License (CC BY). The use, distribution or reproduction in other forums is permitted, provided the original author(s) or licensor are credited and that the original publication in this journal is cited, in accordance with accepted academic practice. No use, distribution or reproduction is permitted which does not comply with these terms. 